\title{
Principles and Practice of Burn Care
}

\section{Sujata Sarabhai}

\section{Edition 2010}

Jaypee Brothers Medical Publishers (P) Ltd. Number of pages: 612

The formulation of strategies for effective burn care in developing countries is a formidable challenge, which only a few bravehearts have accepted. Dr. Sujata Sarabhai, with her new book on "Principles and Practice of Burn Care", offers an outstanding philosophy and protocols for burn care in a country like India, with high population density, illiteracy and poverty, condemning rational burn care to the backburner. Of the 700,000-800,000 annual burn admissions, a paltry amount is treated at state-ofthe-art tertiary care burn centres. Dr. Sarabhai and her co-editors, Prof. V. K. Tiwari, Dr. Arun Goel and Dr. L. C. Gupta, have offered the latest therapeutic strategies and supporting, evidence-based data, all lucidly presented by an eminent group on the forefront of burn care in India. As many as 34 other contributors, each pioneers in their own fields, have poured in their knowledge and lifetime wisdom to transform the 612 pages into an invaluable source of information.

The book is divided into seven exciting sections: Section 1 is devoted to "General Considerations", and contains contributions by the authorities in burn care history, epidemiology and prevention of burns, anatomy of skin, aetiology of burns, microbiology in burns, pathophysiology of burn shock and burn wound, wound healing in burns and immune response to burn injury. In most parts, the current psychological concepts and theories with significant original contributions of recent times have been liberally offered.

Section 2 is on "Early burn care and resuscitation", and the list of contributors is equally imposing. Topics discussed in this section include "Initial evaluation and resuscitation", "respiratory tract burns", "fluidelectrolyte and acid-base balance" and "management of acute renal failure". Easily understandable flow charts and clear and unambiguous guidelines are the hallmark of this section.

Section 3 on "Local care of burn wound" has articles on wound management, surgical excision and grafting, anaesthesia, biological dressings and skin substitutes, skin banking, keratinocyte culture and burn wound infections. A blend of the traditional methods and the very latest in the realm of research adorns this section.

Section 4 deals with "Burns of special types", and has some very informative chapters on electric burns, industrial and chemical burns, radiation burns, friction burns and firework and fire cracker injuries. The problems are typically of the developing world and the solutions offered are affordable, efficient and world-class.

Section 5 is dedicated to "Burns of special sites", and discussed in it are facial burns, hand burns, burns of the perineum and external genitalia, burns in children and burns during pregnancy. Amazing photographs and clear text are the hallmark of this section.

Section 6 on "General management and rehabilitation" is by far the most important part of this book. Issues discussed here are least understood by burn care personnel and are often outsourced to other specialists. It contains chapters like "Multiple organ dysfunction syndromes in burns", "Guidelines for nutritional management", "Postburn sequelae", "Medico-legal aspect of burns" and "Burn disfigurement and physical impairment calculation".

Section 7 deals with "Organization of burn care", and tells the readers how to organize a burn unit and how burn is to be managed in a disaster situation. 
We can only admire and appreciate the dedication, perseverance, tenacity and hard work required in bringing out such a comprehensive book on burn care, which is not only informative but extremely readable as well. The editors and the outstanding group of contributing authors who participated in this edition have to be commended for their extraordinary and timely contributions. Colour photographs and plentiful charts give clear demonstrations of many important points. Jaypee Brothers, Medical Publishers (P) Ltd. have also done a remarkable job in producing this beautiful hardbound book with an appealing book cover and glossy ivory pages offering a perfect contrast to the innumerable colour photographs that adorn them. Although the first edition of this book was released in 2010, delegates of 15th IPRAS had a sneak preview in November 2009. The book has a very crisp foreword penned by Prof. J. L.
Gupta, a doyen of burn care in India and the former Chief of the Department of Burns, Plastic and Maxillofacial Surgery, Safdarjung Hospital, New Delhi, the centre with the largest and busiest burn care unit in the country, and to which the Editor in Chief and her co-editors have been associated with.

Dr. Sujata Sarabhai's "Principles and Practice of Burn Care" is a reference book that every person who is managing burns should read, assimilate and put to practice. Students and doctors alike, who possess this book, will find important practical tips and substantially useful theoretical facts about burn care in it.

\section{Surajit Bhattacharya}

Editor, IJPS surajitbh@yahoo.co.in 